\title{
High-Resolution Gas/Odor Sensors Using High-Frequency Quartz Crystal Microbalance
}

\author{
Severino Muñoz-Aguirre*, Azucena López-Casique, Salvador Alcántara-Iniesta1, \\ Juan Castillo-Mixcóatl, Georgina Beltrán-Pérez and Narcizo Muñoz-Aguirre² \\ Benemérita Universidad Autónoma de Puebla, Facultad de Ciencias Físico Matemáticas, \\ Av. San Claudio y 18 Sur, Col. San Manuel (CU), Puebla, Pue. CP. 72570, México \\ 'Benemérita Universidad Autónoma de Puebla, Centro de Investigación en Dispositivos \\ Semiconductores, 14 Sur y San Claudio (CU), Puebla, Pue, CP. 72570, México \\ ${ }^{2}$ Instituto Politécnico Nacional, Escuela Superior de Ingeniería Mecánica y Eléctrica, Unidad \\ Azcapotzalco, Av. Granjas No. 682, Col. Santa Catarina, CP. 02250, México D.F., México
}

(Received October 1, 2013; accepted January 20, 2014)

Key words: odor sensor, QCM, high frequency, high sensitivity

A study of gas/odor sensors fabricated with a $30 \mathrm{MHz}$ quartz crystal microbalance (QCM) is presented. A problem that appears when working with high-frequency QCM sensors is that the quartz plate becomes thinner and fragile; therefore, the surface structure of the sensing film strongly affects the sensor performance. To solve this problem, thin sensing films were deposited by the ultrasonic atomizer technique. The sensor response to ethanol for different concentrations was stably measured, and a comparison with the response of $20 \mathrm{MHz}$ QCM sensors was performed. It was found that the steady-state response was faster for the $30 \mathrm{MHz}$ QCM sensor owing to the fact that the sensing film is thinner and the ethanol molecules can diffuse more quickly into the sensing film. Furthermore, a sensitivity improvement of approximately two times was achieved.

\section{Introduction}

Quartz crystal microbalance (QCM) gas/odor sensors have been frequently used in arrays for the detection, recognition and discrimination of volatile organic compounds (VOC) in food, beverage and cosmetic industries. ${ }^{(1-3)}$ Although there are a variety of gas/odor sensors, QCM sensors are important since they have a high correlation with the human threshold of detection, ${ }^{(4)}$ they are easily fabricated and no sophisticated equipment is necessary to measure their response (just an oscillation circuit and a conventional frequency counter). Moreover, various selectivities can be achieved just by choosing the

${ }^{*}$ Corresponding author: e-mail: smunoz@fcfm.buap.mx 
appropriate sensing film to be deposited on the QCM electrodes. However, in a variety of applications, it is necessary to measure low concentration levels of odorants or vapors; then, it becomes necessary to raise the sensitivity of the sensors.

To date, there are already some methods of measuring low levels of concentration. The systems are sophisticated and, in some cases, it has been necessary to rearrange the measurement system. ${ }^{(5-7)}$ QCM gas sensors have the advantage that we can improve their sensitivity just by increasing their fundamental oscillation frequency, since the sensitivity depends directly on the square of this parameter according to the Sauerbrey equation. ${ }^{(8,9)}$

Although sensors with very high frequency have been reported up to now, ${ }^{(10,11)}$ there are no reports of sensors constructed with QCM above $20 \mathrm{MHz}$ for HC49U encapsulation, which are extremely cheap and easy to acquire. The reason is that, for higher frequencies, the quartz plate becomes thinner and fragile, and a simple implementation in a cell system is difficult. Moreover, a problem that appears when working with high-frequency QCM is that the surface structure of the sensing film strongly affects the sensor performance. However, with the appropriate film deposition method, this problem can be solved. In the present work, the construction of QCM sensors using $30 \mathrm{MHz}$ crystals with an ethyl cellulose sensing film deposited by the ultrasonic atomizer technique ${ }^{(12,13)}$ is reported.

\section{Experimental Procedure}

The sensors were fabricated using HC49U encapsulation, AT-cut, silver electrode QCMs, and two fundamental oscillation frequencies, 20 and $30 \mathrm{MHz}$ were used. The sensing film was ethyl cellulose (EC, purchased from Sigma-Aldrich) and it was deposited by the ultrasonic atomization technique. ${ }^{(12)}$ This technique uses an ultrasonic atomizer to generate a fine mist of the solution, which contains the sensing film material to be deposited. The mist is transported by an air flow to the QCM electrode. EC is a polymeric material, which can be stably deposited on the QCM electrodes by the ultrasonic atomization technique. Such technique is better than the spray method for the deposition of sensing films since the drop size is smaller and it is possible to obtain a high-quality sensing film surface structure with fewer clumps, which is of crucial importance when the film is deposited on a high-frequency QCM. Sensing films with various thicknesses were deposited for both types of QCM.

The steady-state sensor responses to ethanol were measured. This sample was used since EC has a high response to this vapor. The response was measured using an automated static setup, which is shown in Fig. 1. The sample was injected into the measurement chamber (submerged in a temperature-controlled bath at $25{ }^{\circ} \mathrm{C}$ ), and it was allowed to evaporate until the steady state was reached, while the responses were measured using typical Colpitts oscillation circuits connected to a homemade frequency counter, and the data were stored in a computer. 


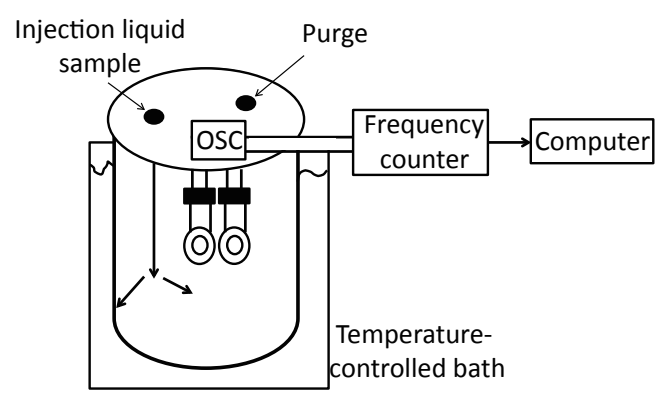

Fig. 1. Sensor response measurement setup.

\section{Results and Discussion}

The QCM sensor principle is based on the resonant frequency shift (sensor response) caused by the adsorption of gas molecules into the sensing film, which is called the mass loading effect. The response is given by the well-known Sauerbrey equation, ${ }^{(8,9)}$ which establishes that the frequency shift is proportional to the amount of adsorbed molecules into the sensing film, as well as to the square of the fundamental oscillation frequency of the QCM. For instance, for an adsorbed mass of $6 \mathrm{ng}$ and an electrode area of $0.28 \mathrm{~cm}^{2}$, a $20 \mathrm{MHz}$ QCM would produce a response of approximately $20 \mathrm{~Hz}$, while a $30 \mathrm{MHz}$ one would produce a response of approx. $44 \mathrm{~Hz}$. Therefore, it can be said that if a sensitivity improvement is required, it would be sufficient just to increase the fundamental frequency of the QCM. The sensitivity increment factor for the same sensing film thickness was found to be 2.25 , when a $30 \mathrm{MHz}$ QCM is used instead of a $20 \mathrm{MHz}$ one. Such value will be valid as long as the sensing film can be stably deposited on the $30 \mathrm{MHz}$ QCM.

An important aspect to consider before and after the sensing film deposition is the study of the $Q$ value, since it determines the oscillation stability of the sensors. In Figs. 2(a) and 2(b), the impedance dependences as a function of the frequency before and after coating for two sensors, 20 and $30 \mathrm{MHz}$, respectively, are shown. For the $20 \mathrm{MHz}$ one, there was a decrease in the $Q$ value of approximately $71 \%$, while in the case of the 30 $\mathrm{MHz}$, such decrease was approximately $65 \%$. It is worth mentioning that it is quite difficult to achieve a highly stable sensing film deposition on the $30 \mathrm{MHz}$ QCM, and in many occasions, the QCM stops its oscillation. The deposition has to be performed carefully in a well-controlled environment and avoiding high air flow rates. In the figures shown above, although the peak seems to be quite rounded for the $20 \mathrm{MHz}$ QCM sensor, its oscillation was more stable than that of the $30 \mathrm{MHz}$ one, whose impedance peak seems more sharpened. However, despite the more unstable oscillation of the $30 \mathrm{MHz}$ QCM sensor, its response can be stably measured although the frequency fluctuations are quite high $( \pm 4 \mathrm{~Hz})$. 


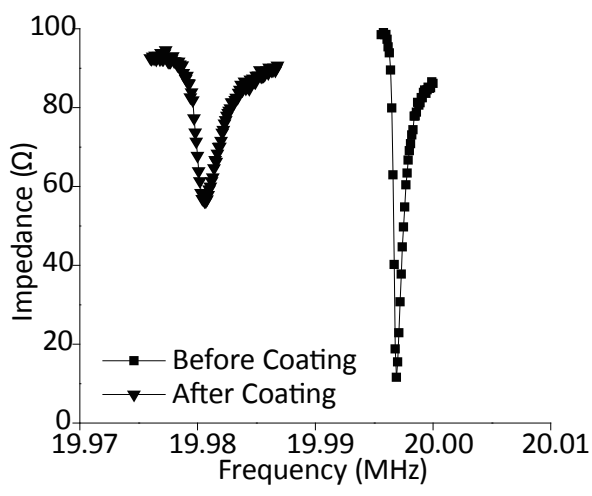

(a)

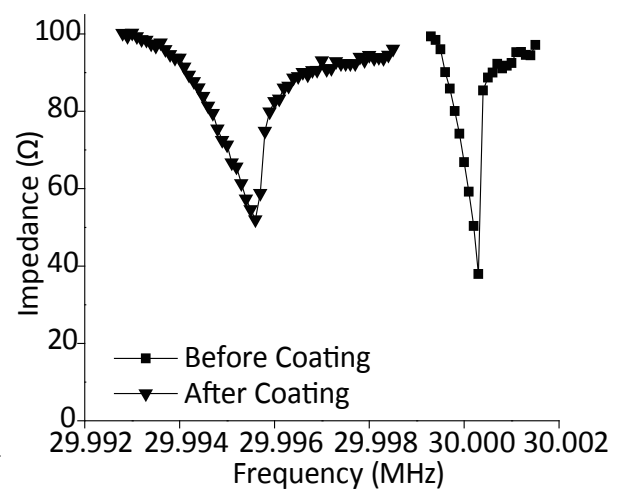

(b)

Fig. 2. QCM impedance curves as a function of frequency for (a) 20 and (b) $30 \mathrm{MHz}$.

To compare the responses of the sensors, response measurements for concentration steps of $4100 \mathrm{ppm}(15 \mu \mathrm{l})$ of ethanol were performed. The responses as a function of time for both 20 and $30 \mathrm{MHz}$ QCM sensors are shown in Figs. 3(a) and 3(b), respectively. In this case, sensing films that caused almost the same frequency shift $(\Delta f=20 \mathrm{kHz})$ were deposited, which means different film thicknesses, i.e., $0.15 \mathrm{~mm}$ for the $20 \mathrm{MHz}$ QCM sensor and $0.06 \mathrm{~mm}$ for the $30 \mathrm{MHz}$ one. The sensing film deposited on the $20 \mathrm{MHz}$ QCM was approximately three times thicker than that deposited on the $30 \mathrm{MHz}$ QCM. It is clear that the sensor response was almost 1.5 times larger for the $30 \mathrm{MHz}$ QCM sensor despite its thinner sensing film. Moreover, the response of this sensor was faster $(\sim 50 \mathrm{~s})$ than that of the $20 \mathrm{MHz}$ QCM ( $250 \mathrm{~s})$ since the ethanol molecules diffuse more quickly inside the thinner sensing film. Therefore, by using the $30 \mathrm{MHz}$ QCM, a larger and faster sensor response was obtained.

In Fig. 4, a comparison between responses as a function of ethanol concentration for both 20 and $30 \mathrm{MHz}$ QCM sensors is shown. In this case, the deposited sensing films had almost the same thickness $\left(d_{\mathrm{f}}=0.025 \mathrm{~mm}\right)$. The response for the $20 \mathrm{MHz}$ QCM sensor below $3000 \mathrm{ppm}$ could not be stably measured, as shown in Fig. 5, where the responses as a function of time are shown for three injections of $2.5 \mu \mathrm{l}$ of ethanol (680 $\mathrm{ppm}$ ). Owing to the frequency fluctuations, a smoothing of the curves was performed. However, only the response of the $30 \mathrm{MHz}$ QCM could be registered since the response of the $20 \mathrm{MHz}$ QCM sensor was too low to be noticed and it was not possible to find a clear trend. Above 3000 ppm, as can be seen in Fig. 4, the sensor response increases linearly and a relationship between responses of around two times was found, which is in agreement with the calculations performed using the Sauerbrey equation. Therefore, it can be said that by using $30 \mathrm{MHz}$ QCM sensors, an improvement of approximately two times in the sensor sensitivity was achieved. 


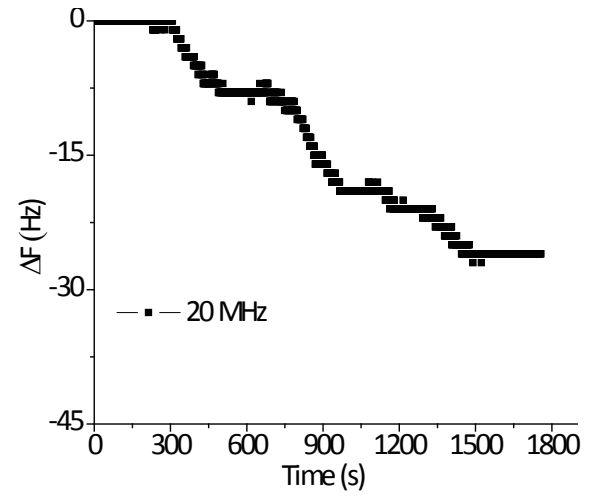

(a)

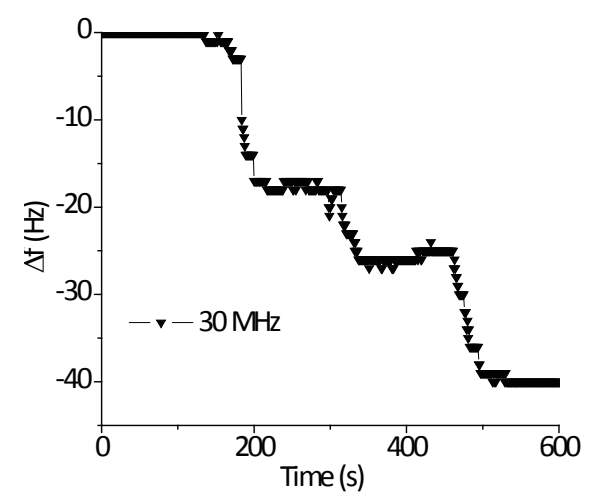

(b)

Fig. 3. Frequency shift as a function of time for (a) 20 and (b) $30 \mathrm{MHz}$ QCM sensors.

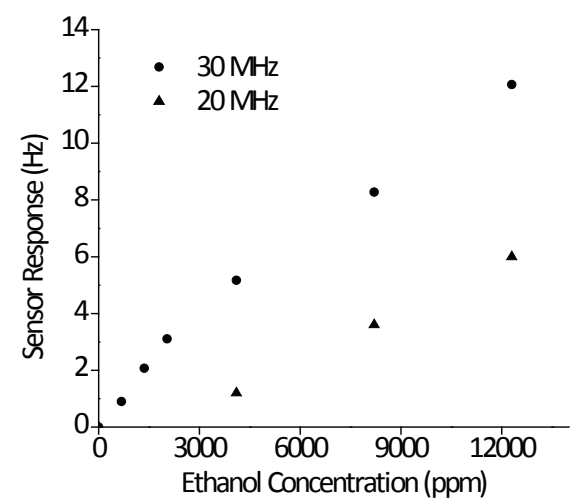

Fig. 4. Responses to ethanol as a function of concentration for 20 and $30 \mathrm{MHz}$ QCM sensors.

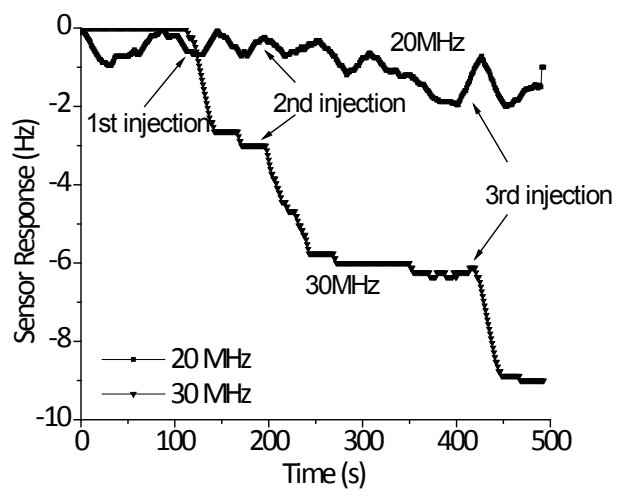

Fig. 5. Comparison of sensor responses to low ethanol concentration exposure for both 20 and 30 $\mathrm{MHz}$ QCM sensors. 


\section{Conclusions}

In this work, a study of gas/odor sensors fabricated with $30 \mathrm{MHz}$ QCM was presented. The EC sensing films were deposited by the ultrasonic atomizer technique to obtain a high-quality sensing film surface, which is desirable to avoid oscillation instability. The sensing film was of high quality since the $Q$ value was not seriously affected, as found from the resonance peak in the impedance curves. Moreover, the sensor responses to ethanol could be stably measured, and from a comparison with the $20 \mathrm{MHz}$ QCM sensor, it was found that the response of the $30 \mathrm{MHz}$ QCM sensor was faster since its sensing film was thinner. Finally, an improvement in the sensitivity of approximately two times was achieved, which is in agreement with the calculations obtained using the Sauerbrey equation. Currently, a study on the improvement in the performance of a sensor array using $30 \mathrm{MHz}$ sensors is under way.

\section{Acknowledgments}

This work was partially supported by the projects CONACyT Jovenes Investigadores 61126 and VIEP-BUAP MUAS-EXC13-I.

\section{References}

1 S. Muñoz-Aguirre, A. Yoshino, T. Nakamoto and T. Moriizumi: Sens. Actuators, B 123 (2007) 1101.

2 T. Nakamoto, K. Aoki, T. Ogi, S. Akao and N. Nakaso: Sens. Actuators, B 130 (2008) 386.

3 M. Peris and L. Escuder-Gilabert: Anal. Chim. Acta 638 (2009) 1.

4 Y. Okahata, O. Shimizu and H. Ebato: Bull. Chem. Soc. Jpn. 63 (1990) 3082.

5 T. Abe and Y. Itasaka: Sens. Actuators, A 188 (2012) 503.

6 J. Ho, Y. K. Fang, K. H. Wu, W. T. Hsieh, C. H. Chen, G. S. Chen, M. S. Ju, J.-J. Lin and S. B. Hwang: Sens. Actuators, B 50 (1998) 227.

7 P. Somboon, B. Wyszynski and T. Nakamoto: Sens. Actuators, B 127 (2007) 392.

8 W. H. King: Anal. Chem. 36 (1964) 1735.

9 A. Arnau and D. Soares: Fundamentals on Piezoelectricity (Springer, Valencia, 2004) p. 27.

10 T. Nakamoto, Y. Suzuki and T. Moriizumi: Sens. Actuators, B 84 (2002) 98.

11 B. P. Stehrer, R. Schwödiauer, S. Bauer, I. M. Graz, P. D. Pollheimer and H. J. Gruber: Procedia Eng. 5 (2010) 835.

12 S. Muñoz-Aguirre, T. Nakamoto and T. Moriizumi: Sens. Actuators, B 105 (2005) 144.

13 B. Wyszynski, A. G. Galvez and T. Nakamoto: Sens. Actuators, B 127 (2007) 253. 